\title{
METODOLOGÍA PARA LA GESTIÓN INTEGRADA E INTELIGENTE DE DESTINOS TURÍSTICOS DE MANABÍ - ECUADOR

\author{
${ }^{1}$ Mabel Font Aranda \\ 2 Joana María Petrus Bey
}

RESUMEN

Objetivo: Sistematizar metodologías que armonicen la gestión integrada e inteligente de destinos turísticos con la información geográfica, para la toma de decisiones oportunas y certeras, aportando a la calidad en el contexto de los territorios turísticos cantonales de la provincia, Manabí - Ecuador.

Metodología/enfoque: Los paradigmas de la gestión de destinos turísticos que concilia todos los participantes, políticas, componentes y partes del sistema; expresado en una gestión integrada. Se engrana, el empleo de las tecnologías de la información y la comunicación en una gestión inteligente, que acoge turistas, comunidades y gestores. Todo en el turismo está atado al espacio y territorio, los sistemas de información que se empleen requieren dar respuesta al "dónde" mediante herramientas de análisis y representación de los lugares.

Originalidad/Relevancia: La secuencia de pasos de varias metodologías y de la teoría sistematizada, enfocan la gestión integrada e inteligente de destinos turísticos, en el tránsito de las partes al todo. En la práctica implica, la participación voluntaria de los empresarios, interesados en la mejora homogénea de la calidad turística en el territorio.

Resultados principales: Un modelo teórico de los conceptos que configuran la comprensión de la gestión integrada e inteligente de destinos y territorios turísticos para la mejora de la calidad. Una metodología reconfigurada a partir de otras, para la puesta en la práctica del modelo teórico.

Contribuciones teóricas / metodológicas: Orientación para la toma de decisiones en los territorios turísticos y de la comprensión por los empresarios y gestores, para revertir la mejora particular de sus negocios, en la mejora homogénea de la calidad turística de los territorios y destinos turísticos de la provincia Manabí.

Palabras clave: Gestión integrada. Turismo inteligente. Gestión de destinos. Mejora de la calidad. Territorios turísticos.

Received on August 07th, 2020 Approved on December 23th, 2020 (c) (1) ()

\section{(APA)} Font Aranda, M., \& Petrus Bey, J. (2021). Metodología para la gestión integrada e inteligente de destinos turísticos de Manabí - Ecuador. International Journal of Professional Business Review, 6(1), 1-15. doi: http://dx.doi.org/10.26668/businessreview/2021.v6i1.208 


\title{
METHODOLOGY FOR INTEGRATED AND SMART TOURISM DESTINATION MANAGEMENT IN MANABÍ - ECUADOR
}

\begin{abstract}
Objective: Systematize methodologies that harmonize integrated and smart tourist destinations management with geographic information, for making timely and accurate decisions, contributing to quality in the context of the cantonal tourist territories of the province, Manabí - Ecuador.

Methodology / approach: The paradigms of the management of tourist destinations that reconcile all the participants, policies, components and parts of the system; expressed in an integrated management. The use of information and communication technologies is geared towards smart management, that envolve tourists, communities and managers. Everything in tourism is tied to space and territory, the information systems used require a response to the "where" through analysis and representation tools of places.
\end{abstract}

Originality / Relevance: The sequence of steps of various methodologies and of the systematized theory, focus the integrated and smart tourim destination management, in the transit from the parts to the whole. In practice, it implies the voluntary participation of businessmen, interested in the homogeneous improvement of tourist quality in the territory.

Main results: A theoretical model of the concepts that make up the understanding of the integrated and smart tourism destination management and territories for the improvement of quality. A reconfigured methodology from others, for putting the theoretical model into practice.

Theoretical / methodological contributions: Guidance for decision-making in tourist territories and understanding by entrepreneurs and managers, to reverse the particular improvement of their businesses, in the homogeneous improvement of the tourist quality of the territories and tourist destinations of the Manabi province.

Keywords: Integrated management. Smart tourism. Destination management. Quality improvement. Tourist territories. 


\section{METODOLOGIA PARA A GESTÃO INTEGRADA E INTELIGENTE DOS DESTINOS TURÍSTICOS EM MANABÍ - EQUADOR}

\section{RESUMO}

Objetivo: Sistematizar metodologias que harmonizem a gestão integrada e inteligente dos destinos turísticos com a informação geográfica, para tomar decisões oportunas e precisas, contribuindo para a qualidade no contexto dos territórios turísticos cantonais da província de Manabí - Equador.

Metodologia / abordagem: Paradigmas de gestão de destinos turísticos que reconciliam todos os participantes, políticas, componentes e partes do sistema; expressa em uma gestão integrada. A utilização das tecnologias de informação e comunicação está orientada para uma gestão inteligente, que acolhe turistas, comunidades e gestores. Tudo no turismo está ligado ao espaço e ao território, os sistemas de informação utilizados exigem uma resposta ao "onde" através de ferramentas de análise e representação dos lugares.

Originalidade / Relevância: A sequência de passos de várias metodologias e teoria sistematizada, centra-se na gestão integrada e inteligente dos destinos turísticos, no trânsito das partes para o todo. Na prática, implica a participação voluntária de empresários, interessados na melhoria homogênea da qualidade turística do território.

Principais resultados: Um modelo teórico dos conceitos que configuram a compreensão da gestão integrada e inteligente de destinos e territórios turísticos para a melhoria da qualidade. Uma metodologia reconfigurada de outras, para colocar em prática o modelo teórico.

Contribuições teórico-metodológicas: Orientação para a tomada de decisão em territórios turísticos e compreensão por empresários e gestores, para reverter a melhoria particular dos seus negócios, na melhoria homogénea da qualidade turística dos territórios e destinos turísticos da cidade. Província de Manabi.

Palavras-chave: Gestão integrada. Turismo inteligente. Gerenciamento de destino. Melhoria da qualidade. Territórios turísticos. 


\section{INTRODUCCIÓN}

En la actualidad la gestión de destinos turísticos se encuentra en un foco de transformación, debido a la incidencia de las tecnologías de información y comunicación (TIC), así como la participación de los entes involucrados, aspectos que adquieren importancia clave para su calidad e imagen. Coinciden Gomez-Oliva, Alvarado-Uribe, ParraMeroño, \& Jara (2019); García-Milon, JuanedaAyensa, Olarte-Pascual, \& Pelegrín-Borondo (2020), Kiatkawsin, Sutherland, \& Lee (2020), (Shen, Sotiriadis, \& Zhang, 2020:15); que hay una creciente necesidad de nuevas soluciones TIC para destinos turísticos inteligentes, porque los nuevos visitantes digitales han evolucionado a inteligentes y utilizan las nuevas tecnologías para planificar y disfrutar de viajes y visitas; intercambian datos, interactúan y co-crean experiencias.

La inclusión y la participación social constituyen elementos intrínsecos a los espacios y destinos turísticos, porque de esa forma se vinculen entre sí (Salessi, 2017). También, existe un gap muy importante en el sector turístico donde se implantan Sistemas de Gestión de la Calidad en base a normas específicas para cada uno de los subsectores que lo componen (Álvarez, Del Río, Durán y Urbano, 2017:12). El mayor de los retos es afrontar con éxito el cambio de mentalidad en la gestión de los destinos (Ivars-Baidal, Celdrán-Bernabeu, Mazón \& PerlesIvars, 2017). Hilvanar todos los aspectos mencionados, constituye un desafío que tienen que asumir los gestores de los diferentes territorios y destinos turísticos.

Desde hace décadas se destaca a nivel mundial, el Programa Integral de Calidad Turística Española (PICTE 2000-2006) y la expresión actual del Sistema Integral de Calidad Turística Española en Destinos (SICTED). Constituido en proyecto y modelo, "surge de la necesidad de dar una solución capaz de gestionar íntegramente la calidad en un destino turístico. Este enfoque implica que no sólo se aborda la calidad para un oficio o una empresa en concreto, sino para todos los servicios que se ofrecen en un destino turístico" (Secretaría de Estado de Turismo, 2019a:3). También en España, con el inicio del Plan Nacional e Integral de Turismo (2012-2015), se han desarrollado los destinos turísticos inteligentes (DTI), donde se diseñan una serie de líneas de trabajo para su formalización, en colaboración con la Sociedad
Estatal para la Gestión de la Innovación y las Tecnologías Turísticas, S.A. (SEGITTUR) (CeldránBernabeu, Mazón \& Sánchez, 2018).

Los principios básicos de las experiencias españolas referidas, pueden ser aplicados en otros entornos de Latinoamérica, específicamente de Ecuador y en particular de la provincia Manabí. Determinados aspectos que caracterizan a SICTED y DTI - SEGITTUR, con una configuración para tal contexto. En correspondencia, se busca preparar una metodología que favorezca estratégicamente, la gestión integrada e inteligente de destinos turísticos utilizando la información geográfica.

Constituyen problemas de la gestión para el desarrollo turístico en la provincia Manabí: inequidad y debilidad de los servicio turísticos, la presión de la contaminación, extracción de recursos y crecimiento de la población en la franja costera, carencia de certificación de calidad en servicios de alojamiento y alimentación, insuficiente fiscalización y regulación de actividades turísticas, limitada innovación en agencias de viajes y operadoras turísticas, sector informal que compite con actividades legalmente constituidas, desarticulación entre diferentes niveles de gobierno, problemas de carácter social con necesidades básicas insatisfechas, separación entre objetivos de desarrollo y realidad del territorio (Proaño y Ramírez Pérez (2017).

Entre las falencias prioritarios que enumera la Agenda Zonal 4 (2017-2020) está el débil aprovechamiento de las potencialidades y diversidad de recursos por parte del sector de turismo. La realidad de la provincia Manabí exige fomentar a nivel local la inversión y el emprendimiento, aprovechando los atractivos turísticos de la Zona, mediante el mejoramiento de infraestructura y servicios turísticos, con un enfoque de sostenibilidad ambiental y articulación de los diferentes actores institucionales, empresariales y comunitarios (SENPLADES 2019:33).

La interpretación de los aspectos ya señalados sustenta la formulación del problema: ¿Cómo contribuir a la gestión de destinos que aporte a la calidad en el contexto de los territorios turísticos cantonales de la provincia Manabí - Ecuador? 
El objeto de investigación se centra en la gestión de destinos turísticos y el campo de acción específico es una metodología que armonice la gestión integrada inteligente de destinos turísticos y la información geográfica, para la mejora de la calidad. El objetivo general es sistematizar metodologías seleccionadas que armonicen estos criterios de gestión para la toma de decisiones oportunas y certeras, aportando a la calidad en el contexto de los territorios turísticos cantonales de la provincia, Manabí - Ecuador (Figura 1).

Figura 1. Provincia Manabí, Ecuador

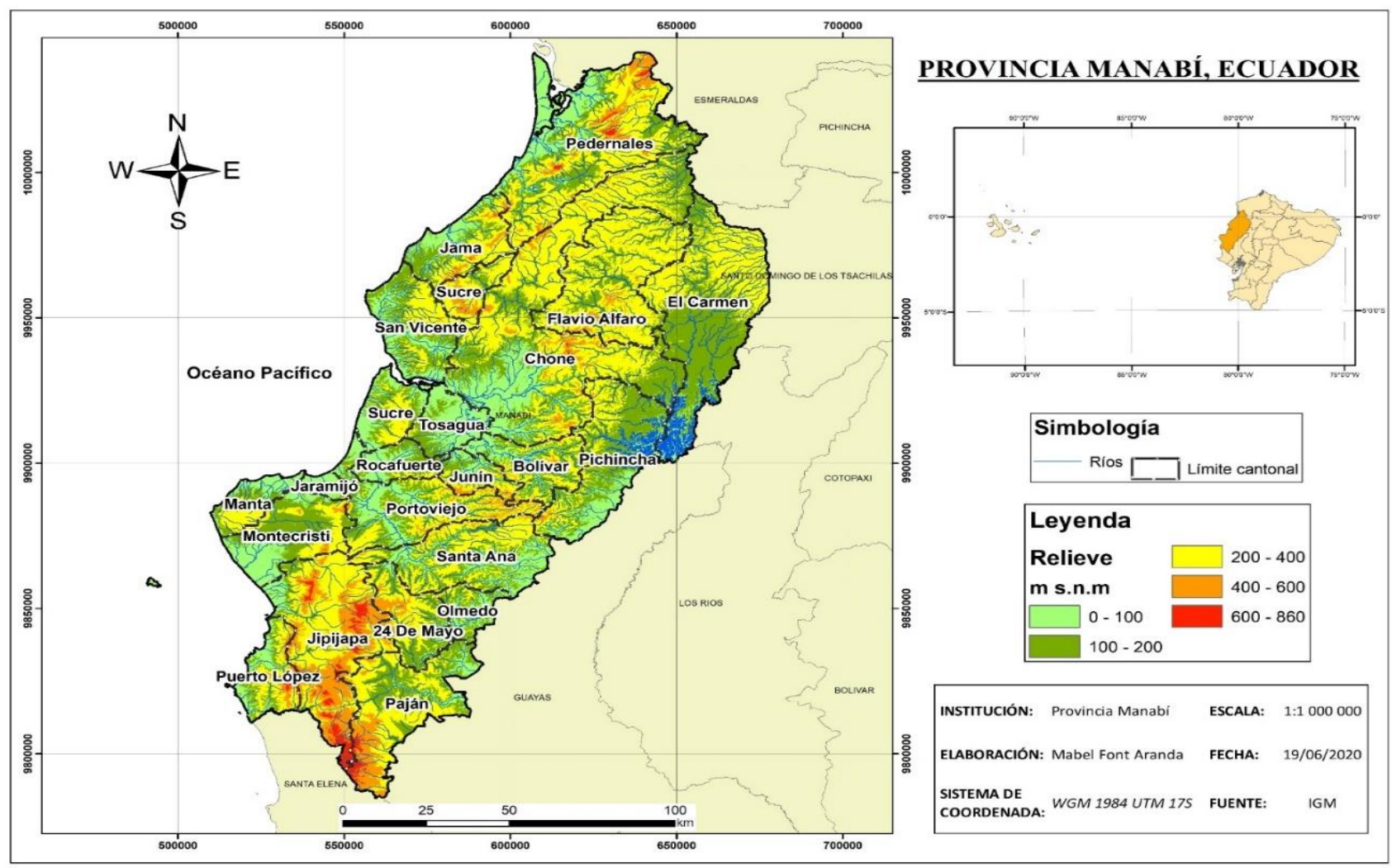

Fuente: Elaboración propia a partir de las capas del Instituto Geográfico Militar de Ecuador

\section{La gestión integrada e inteligente de destinos y territorios turísticos}

La gestión relacionada con el turismo está inevitablemente vinculada a la noción geográfica, de una u otra forma. Lo anterior se debe a que la actividad se gesta, dado el movimiento de personas desde sus lugares habituales. Todo, por la motivación de experimentar, determinadas sensaciones en otros contextos, donde existen atractivos y servicios que sustentan los intereses diversos. Señala la OMT (2019a:10) que un destino turístico es un espacio físico, con o sin una delimitación de carácter administrativo o analítico, en el que un visitante puede pernoctar. Es una agrupación (en una misma ubicación) de productos y servicios, y de actividades y experiencias, en la cadena de valor del turismo, y una unidad básica de análisis del sector. Un destino incorpora a distintos agentes y puede extender redes hasta formar destinos de mayor magnitud. Es además inmaterial, con una imagen y una identidad que pueden influir en su competitividad en el mercado

Un Destino Turístico Inteligente (DTI) puede ser entendido como "un espacio turístico innovador, accesible para todos, consolidado sobre una infraestructura tecnológica de vanguardia que garantiza el desarrollo sostenible del territorio, facilita la interacción e integración del visitante con el entorno e incrementa la calidad de su experiencia en el destino y la calidad de vida de los residentes" (Secretaría de Estado de Turismo - SEGITTUR, 2018). En este contexto, señala Salessi (2017) que cuatro conceptos son ejes centrales tales como: la innovación, la tecnología, la accesibilidad universal y la sostenibilidad, que constituyen los requisitos estructurales para la creación de nuevos modelos de destinos turísticos inteligentes (DTI), agregan la

Intern. Journal of Profess. Bus. Review; São Paulo V.6 N.1 2021, pp. 1-15, Jan/Dec.2021 
gobernanza, la Secretaría de Estado de Turismo SEGITTUR (2018), OMT (2019b:11). Un destino turístico inteligente, también depende de turistas inteligentes. Además, las empresas deberían colaborar no solo con otras empresas sino también con las organizaciones de gestión de destinos Kiatkawsin, Sutherland, \& Lee (2020:11).

La gestión de destinos inteligentes, tiene su antecedente en la gestión de ciudades inteligentes, los pilares o dimensiones de estas, también sostienen a los destinos: movilidad, gobernanza, poblaciones, calidad de vida, economía y ambiente (Sigalat-Signes, Calvo-Palomares, Roig-Merino \& García-Adán, 2020:103). En la gestión de destinos turísticos se destaca la organización de gestión/marketing de destino (OGD/OMD) conformada por diversas autoridades, agentes y profesionales, en una configuración colectiva. Las organizaciones de gestión de destinos (OGD), incluyen diferentes estructuras y la tendencia es hacia las alianzas público-privada, y su función esencial es iniciar, coordinar y gestionar ciertas actividades, tales como la aplicación de políticas turísticas, la planificación estratégica, el desarrollo de productos, la promoción y el marketing y el trabajo de las convenciones (OMT, 2019b).
En este ámbito, se asume la premisa planteada por Salessi (2017) la inclusión y la participación social constituyen elementos intrínsecos a los espacios y destinos turísticos, para que de esa forma inteligencia e inclusión se vinculen entre sí. Estos aspectos son considerados en la gestión integrada del destino. Continúa Salessi (2017) expresando que los destinos turísticos inteligentes se nutren de datos, gobierno abierto, y participación ciudadana configurados en una matriz altamente flexible y dinámica. De acuerdo con Celdrán-Bernabeu, Mazón, Ivars-Baidal, \& VeraRebollo (2018:112), el concepto inteligencia se centra en la capacidad técnica para ofrecer los servicios adecuados a los usuarios, también se relaciona con la experiencia o el resultado de una experiencia. Además, los residentes pueden disfrutar de muchos de los servicios de alto valor añadido en destinos inteligentes, poniendo a su disposición servicios públicos más eficientes y accesibles, y mejorando su conocimiento y uso (incluido el disfrute del patrimonio y de las atracciones turísticas) (Crespo, Gutiérrez \& Sánchez, 2019:79). La figura 2 sintetiza las lecturas anteriores e incorpora los rasgos comunes de los territorios inteligentes declarados por López de Ávila, (2015:112).

Figura 2. Rasgos comunes a los territorios inteligentes

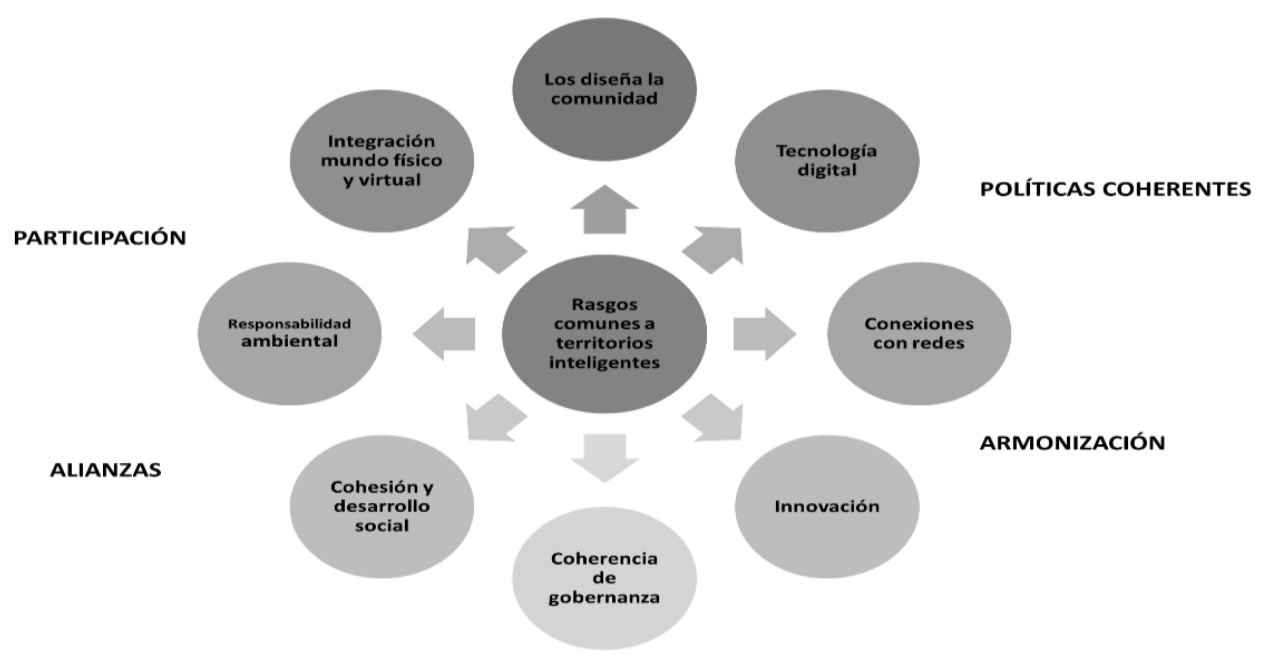

Fuente: Elaboración propia a partir de los diferentes autores.

Se promoverá el uso compartido de buenas prácticas con otros destinos turísticos, a fin de obtener áreas de mejora -superadoras- en cuanto a la prestación de productos y servicios turísticos de calidad, y la detección de problemáticas comunes entre municipios o comunidades, construyendo modelos replicables que actúen como guía de referencia en otras ciudades o destinos interesados en esta visión de proyecto colectivo (Salessi, 2017). El valor del destino inteligente -concluyen Crespo, 
Gutiérrez \& Sánchez (2019:88)- se expresa en un fenómeno complejo de perspectiva multidimensional, que exige examinar la influencia de las actitudes y comportamientos de los diversos actores o stakeholders del territorio -residentes o turistas, entre otros.

\section{Calidad de un destino o territorio turístico}

La calidad en el ámbito del turismo adquiere diversos matices y connotaciones, su abordaje desde los tipos de empresas turísticas o del destino, está sujeto a estándares o a criterios de percepción. Luis del Campo et al. (2017:98) asevera que en relación con la calidad de las empresas que prestan el servicio, cabe destacar existen diversos modelos y sistemas genéricos de calidad centrados en los procesos (normativa ISO 9000 y 9001). No obstante, en función de la diversidad de perfiles e intereses, la valoración que se realice de la calidad en el turismo cambiará, y adquirirá diferentes patrones.

En la búsqueda por mejores alternativas, recibe importancia el vínculo cada vez mayor con el cliente externo e interno. A nivel del destino turístico, la calidad se complejiza, ya que además de cómo se manifieste el sector empresarial de forma individual o colectiva, se agrega el sentir de la comunidad, el estado del medio ambiente, de los atractivos turísticos que soportan las ofertas y actividades.

La calidad del servicio es un factor determinante de la satisfacción del cliente (Su, et al. 2016:83), según sea percibida se considera el grado y la dirección de discrepancia entre las percepciones y expectativas de los consumidores (Parasuraman, et al. 1988:17). La satisfacción del cliente es la variable principal respecto la entrega de valor superior, es factor destacado para retener un cliente fiel; se deriva de la interacción cliente empresa, de la construcción de una mejor experiencia y satisfacción del cliente (González-Mansilla et al. 2019:51). En consideraración a la heterogeneidad del visitante, cada vez más el valor co-creación con los clientes se ha convertido en un tema relevante, tanto en el ámbito académico y de gestión (Dolan \& Kemper, 2019:36; González-Mansilla et al. 2019:51).

Con frecuencia se examina la relación entre calidad del servicio y comportamiento del cliente, pero no se ha examinado como consecuencia el bienestar subjetivo del cliente (Su, et al. 2016:83). Este propio autor, enfatiza que "mientras que la calidad del servicio es una evaluación global de un rendimiento, calidad de la relación es una orientación estratégica que se centra en la mejora de relaciones con los clientes como un enfoque estratégico". Resalta a Lages et al. (2005) quien representa la calidad de la relación como la cantidad de intercambio de información, la calidad de la comunicación, la orientación a largo plazo, y la satisfacción con una relación. De lo planteado por los autores antes citados, se precisa lo mucho que aporta la gestión inteligente del destino y los territorios turísticos.

La calidad de un destino turístico es el resultado de un proceso que implica la satisfacción de todas las necesidades, requisitos y expectativas del consumidor con respecto a los productos y servicios turísticos, a un precio aceptable, de conformidad con unas condiciones contractuales mutuamente acordadas, y factores subyacentes implícitos, tales como la seguridad, la higiene, la accesibilidad, la comunicación, la infraestructura y las instalaciones y servicios públicos. Considera, además, aspectos relacionados con la ética, la transparencia y el respeto por el entorno humano, natural y cultural. La calidad, por ser uno de los motores clave de la competitividad turística, es también una herramienta profesional para los proveedores turísticos a efectos organizativos, operativos y de imagen (OMT, 2019b:15). Todos estos aspectos, constituyen datos expresables en un sistema de información georeferenciada y esencial para la gestión inteligente de territorios y destinos. La sistematización de la calidad contempla tres niveles de actuación: organizativo, operativo y de reconocimiento (OMT, 2015:16). En sintonía, SICTED es un proyecto de calidad de destinos turísticos, direccionado a mejorar la experiencia y satisfacción del turista. Se basa en el trabajo conjunto de los gestores del destino y los empresarios turísticos. Plantean Ivars-Baidal y Vera Rebollo (2019:10) que, en la evolución reciente de la planificación turística en España, SICTED ha fundamentado la calidad integral, pero constituye novedad las iniciativas de los Destinos Turísticos Inteligentes planteados por SEGITTUR, proyectos no interconectados. Los objetivos de SICTED son crear una estructura permanente de gestión e impulso a la calidad, aplicar estándares básicos de calidad y reconocer el esfuerzo de los participantes (Secretaría de Estado de Turismo, 2019a:4) (Tabla 1). 
Tabla 1. Interrogantes clave sobre Sistema Integral de Calidad Turística en Destinos

\begin{tabular}{|l|l|}
\hline Interrogante & Descripción \\
\hline ¿Qué? & Proyecto, modelo, enfoque, metodología \\
\hline ¿Quiénes? & Gestores, empresas, agentes y prestadores de servicios turísticos (Voluntario) \\
\hline ¿Cómo? & Integrado, participativo, inteligente \\
\hline ¿Para qué? & Mejorar la calidad, experiencia y satisfacción del turista \\
\hline
\end{tabular}

Fuente: Elaboración propia a partir de Secretaría de Estado de Turismo (2019a - 2019b).

Esta metodología se basa en el concepto de itinerario de consumo, en el que un mismo turista es atendido por los profesionales de distintos oficios, debiendo percibir un nivel de atención equilibrado en el destino turístico (Secretaría de Estado de Turismo, 2019a:3), por eso debe cumplir ciertas características (tabla 5).

Tabla 2. Las características de SICTED

\begin{tabular}{|l|l|}
\hline Características & Descripción \\
\hline $\begin{array}{l}\text { Integrador y } \\
\text { participativo }\end{array}$ & $\begin{array}{l}\text { Todos los agentes turísticos del destino se unen para la mejora de la prestación de } \\
\text { servicios y la satisfacción de turistas y comunidades. }\end{array}$ \\
\hline $\begin{array}{l}\text { Creador de ventajas } \\
\text { competitivas. }\end{array}$ & $\begin{array}{l}\text { Facilita el seguimiento y control para la satisfacción del visitante en la mejora } \\
\text { permanente. }\end{array}$ \\
\hline Estructurado & Se estructura en tres ejes: gestor, manual de buenas prácticas y cuadro de control. \\
\hline Flexible & $\begin{array}{l}\text { Dada la diversidad de unidades territoriales, tipologías y modalidades de turismo } \\
\text { en el destino, las configuraciones disímiles exigen maleabilidad. }\end{array}$ \\
\hline Integral & Incide en todos los componentes del sistema turístico, \\
\hline Impulsor de otros & Estimula la inserción de diferentes sistemas informáticos y tecnológicos que se \\
productos tecnológicos & revierta en la mejora continua de la calidad. \\
\hline Orientado a resultados & A través de indicadores se miden los resultados. \\
\hline $\begin{array}{l}\text { Organizado por } \\
\text { procesos }\end{array}$ & $\begin{array}{l}\text { Cada tipología de gestión sigue una secuencia lógica de actividades en } \\
\text { subprocesos. }\end{array}$ \\
\hline
\end{tabular}

Fuente. Elaboración propia a partir de Secretaría de Estado de Turismo (2019a).

En la metodología de SICTED, es clave el conjunto de buenas prácticas voluntarias que aplican a más de 35 oficios del sector Turístico (Secretaría de Estado de Turismo (2019c:10). Estás prácticas están registradas en manuales, y tiene dos funciones: una individual servir de instrumento a las empresas para instaurar un sistema de gestión de la calidad; y una colectiva: proporcionar indicadores para el plan de mejora del destino, según el grado de cumplimiento de estos (Corral-Marfil. 2012:9; Mellén Madruga y Soler Reglí, 2018).

Los manuales de buenas prácticas son el eje para la mejora de SICTED, se estructuran en capítulos y a su vez en módulos. Existen cuatro capítulos que se organizan de lo general a lo particular hasta el capítulo III: Intersectorial, Actividad principal y Actividad complementaria. El cuarto capítulo Avanzadas que incluye aspectos transversales como 
la sostenibilidad e innovación y tiene carácter voluntario. Todo el sistema se organiza en ciclos.

Acerca del control de la calidad, precisa CorralMarfil (2012:11) que, supone indicadores de la situación y la evolución de la calidad del destino. Indicadores referidos a subsectores turísticos (alojamiento, restauración), otros subsectores (comercio, sanidad) y atributos compartidos por diversos agentes, pero percibidos globalmente por los visitantes (limpieza, seguridad ciudadana, infraestructuras). Toda la metodología está soportada en Sistemas de Información con expresión geográfica.

\section{Concepción teórico metodológica para la gestión integrada e inteligente de destinos y territorios turísticos}

La concepción teórico metodológica construida, se muestra en la figura 3, y sistematiza todos los preceptos teóricos previamente abordados. La lectura realizada desde la base, permite interpretar diferentes criterios.

Figura 3. Concepción teórico metodológica para gestión integrada e inteligente de destinos y territorios turísticos.

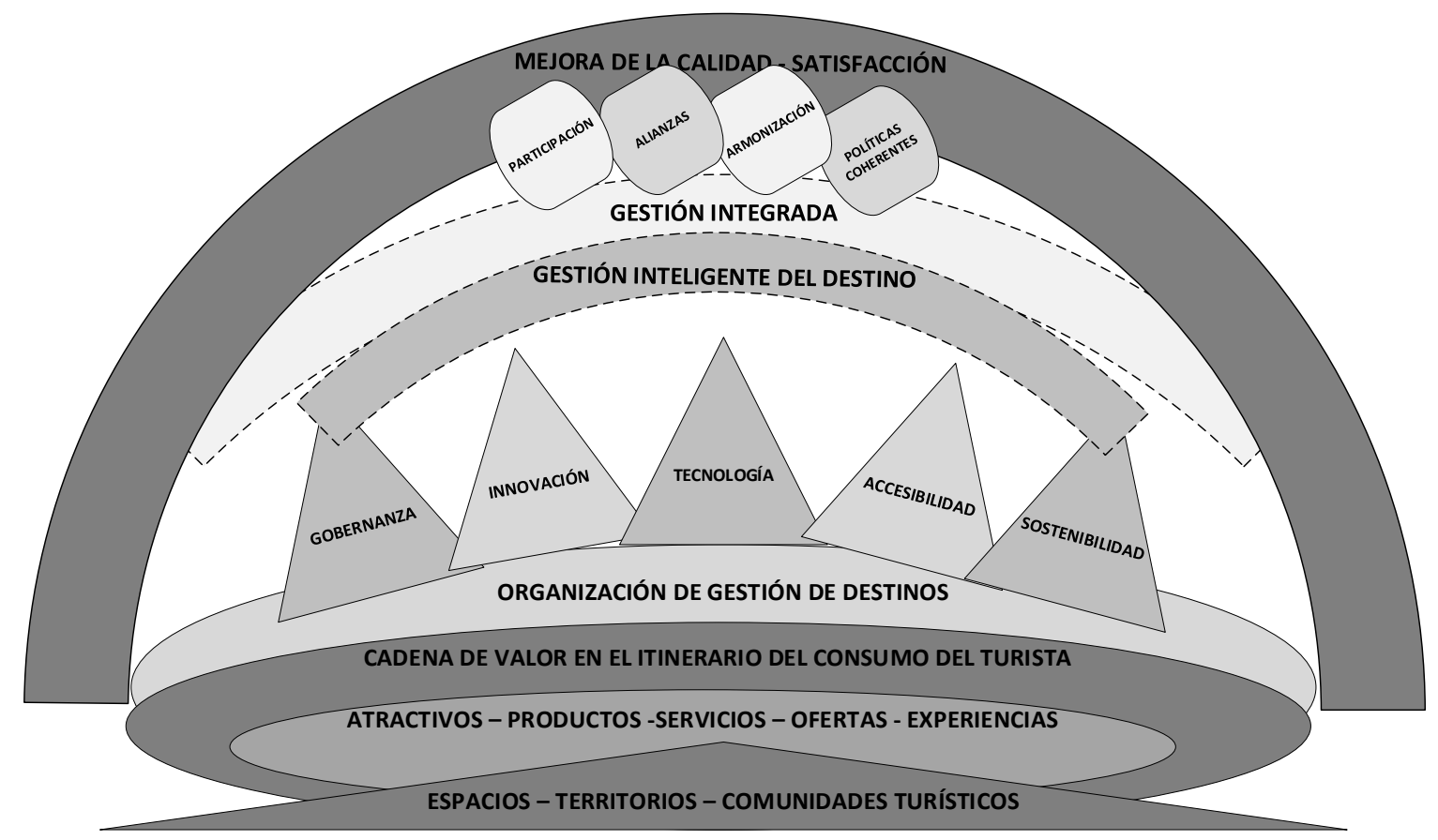

Fuente: Elaboración propia.

1-El turismo es una actividad que se erige en espacios y territorios, donde se asientan los genuinos propietarios que son las comunidades.

2- En los espacios y territorios turísticos existen recursos y atractivos, en torno a los cuales se configuran productos, ofertas, servicios $y$ experiencias; que motivan el desplamientos, porque son inamovibles.

3- Alrededor de ese engranaje se desarrolla el itinerario del consumo del turista, el cual todo el tiempo compra servicios y adquiere experiencias.

4- Indivisiblemente lo anterior, debería gestarse en una Organización de Destinos Turísticos.
5- Para el logro de una gestión inteligente de destinos turísticos, los pilares son: gobernanza, innovación, tecnología, accesibilidad y sostenibilidad.

6- La gestión integrada se logra, si hay participación de las partes implicadas, alianzas entre organizaciones y empresas públicas, privadas, comunitarias, la armonización de los diferentes procesos a partir de políticas coherentes.

7- Como resultado, la mejora de la calidad engloba cada una de las partes y por tanto la satisfacción de todos los involucrados.

Desde la mirada de diferentes autores se explica la concepción teórico metodológica. El contenido 
original, creado por las comunidades de residentes, es más atractivo para el nuevo visitante digital, el patrimonio inmaterial tiene un alto valor para que los pueblos construyan una marca local; las comunidades de vecinos tienen un gran interés en participar y colaborar con su municipio (Gómez-Oliva, Alvarado-Uribe, Parra-Meroño, \& Jara, 2019:26). El estudio desarrollado por Pai, Liu, Kang, \& Dai (2020:3) muestra que la mayoría de los turistas tienen una intención positiva de utilizar las tecnologías de turismo inteligente y que las organizaciones de gestión de destinos pueden crear actividades y experiencias específicas para los turistas mediante estas, especialmente en términos de personalización. Un enfoque de gestión inteligente conducirá al desarrollo y crecimiento de la industria turística en el territorio, con externalidades positivas a través de la creación de empleos y riqueza para la población local (Crespo, Gutiérrez \& Sánchez, 2019:88).

Respecto la gestión inteligente e integrada, el desarrollo de destinos inteligentes aún se encuentra en la fase inicial, la adopción de estrategias tecnológicas pertinentes para alinearlo con los requisitos estipulados por el modelo inteligente va unida a la dimensión ciudadano y su participación como un pilar (Sigalat-Signes, Calvo-Palomares, RoigMerino \& García-Adán, 2020:103). Las tecnologías inteligentes incluyen una variedad de tecnologías informáticas y de la información, como son: Internet de las Cosas, Tecnología de computación en la nube, Inteligencia artificial, Tecnología de comunicación móvil, Aplicaciones y dispositivos móviles, Big Data, Conexión entre Wi-Fi y otras redes, Realidad virtual y aumentada, Robot de chat inteligente, Dispositivos portátiles, Red de balizas (Shen, Sotiriadis, \& Zhang, 2020). El núcleo del desarrollo del sistema de gestión del turismo inteligente es la base de datos. Todos los datos del proceso de funcionamiento del sistema, se gestionan mediante el almacenamiento de datos y la extracción de la base de datos (Gao, \& Li, 2020:3) . Los responsables de la gestión turística son los encargados de incrementar el desarrollo de destinos inteligentes, desarrollando destinos basados en la innovación, la tecnología, la accesibilidad y la sostenibilidad (Liberato, P., Alen, \& Liberato, D. 2018:21). Un canal de comunicación innovador que construya experiencias ágiles, aumente la difusión cultural y validez de la viabilidad de crear un ciclo sostenible de contenidos a través de la cooperación de visitantes y prosumidores digitales (Gómez-Oliva, Alvarado-Uribe, Parra-Meroño, \& Jara, 2019:2).
$Y$ puesto que, todo en turismo se asienta en espacio y territorios a partir de la movilidad del visitante que consume servicios, productos y experiencias locales; se coincide con Afnarius, Syukur, Ekaputra, Parawita, \& Darman (2020:4) cuando señalan que requiere el uso de Tecnología Inteligente a través de la aplicación de Internet y Geoinformática. También, tecnologías adicionales, como el sistema de posicionamiento global (GPS), la teledetección, los servicios web y los servicios basados en la ubicación. Sin embargo, los componentes más importantes incluyen datos geoespaciales y Sistemas de Información Geográfica.

En lo que concierne a la mejora de la calidad y satisfacción, el uso adecuado de tecnologías inteligentes puede contribuir a experiencias atractivas y memorables que generen beneficios para todos los actores en el marco de la gestión del turismo inteligente (Shen, Sotiriadis, \& Zhang, 2020:15). Las personas pueden visitar virtualmente los lugares y participar en actividades con sus teléfonos inteligentes antes de visitar el lugar. Las fuentes de información turística inteligente deben ser informativas, accesibles, interactivas y flexibles; ya que estos atributos están dirigidos a la satisfacción del viajero (Ranasinghe, Danthanarayana, Ranaweera, \& Idroos, 2020:10). El turismo inteligente involucra todos los aspectos del turismo, incluidos el transporte, el alojamiento y las atracciones. Cuando los turistas tienen emociones y actitudes positivas hacia las tecnologías de turismo inteligente, su experiencia en el destino quedará satisfecha. Como resultado, la satisfacción de viajar produce felicidad turística (Pai, Liu, Kang, \& Dai, 2020:3).

\section{Metodología para la gestión integrada e inteligente de destinos turísticos, Manabí - Ecuador}

Refiriéndose a resultados de investigación, Barreras Hernández (2004) decía que una metodología es el conjunto de métodos, procedimientos, técnicas, que, regulados por determinados requisitos, permiten ordenar el pensamiento y los modos de actuación para obtener y descubrir nuevos conocimientos teóricos o la solución de problemas de la práctica. La metodología que se presenta es un aporte teórico-práctico. Refleja una secuencia de pasos con el objetivo de contribuir a la gestión integrada e inteligente de destinos y territorios turísticos en la provincia de Manabí - 
Ecuador. La necesidad de un cambio de gestión en tales contextos, se expresan en la figura 4.

Figura 4. Planteamiento del problema para una gestión integrada e inteligente en Manabí, Ecuador.
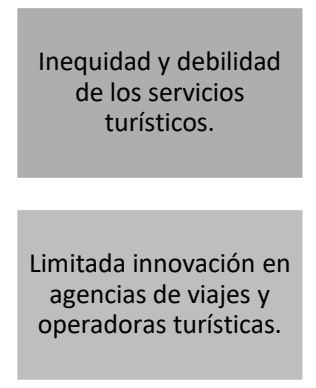
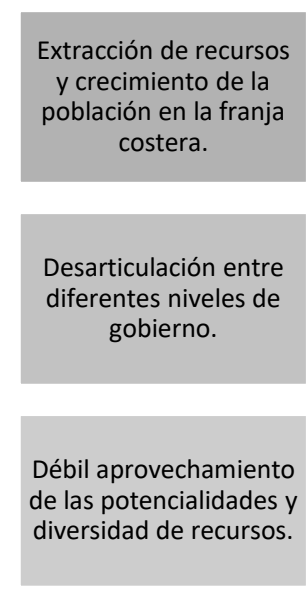

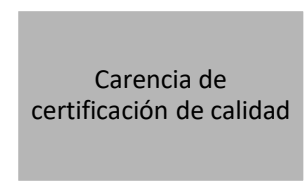

Problemas de carácter social con necesidades básicas insatisfechas.

Necesidad de fomentar a nivel local la inversión y el emprendimiento

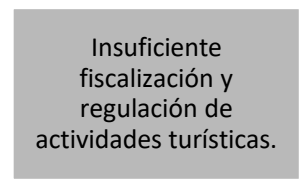

Separación entre objetivos de desarrollo y realidad del territorio.

Fuente: Elaboración propia a partir de Proaño y Ramírez Pérez (2017) y SENPLADES. (2019).

Se muestra un conjunto de métodos, procedimientos, técnicas, de acuerdo con requisitos, para ordenar los modos de actuación en la solución de problemas prácticos ya declarados, respecto al desarrollo turístico de los espacios y territorios propios del presente campo investigación, su implementación incidirá en la transformación de estado actual e incrementará la satisfacción del visitante y las comunidades.

Tabla 3. Elementos de la metodología.

\begin{tabular}{|c|c|}
\hline Elementos & Descripción \\
\hline 1- Objetivo que se pretende alcanzar & $\begin{array}{l}\text { Contribuir a la gestión integrada e inteligente para la } \\
\text { toma de decisiones oportunas y certeras, aportando } \\
\text { a la calidad en el contexto de los territorios turísticos } \\
\text { cantonales de la provincia, Manabí- Ecuador. }\end{array}$ \\
\hline $\begin{array}{l}\text { 2- Objeto de estudio, conceptos y categorías que lo } \\
\text { describen y explican }\end{array}$ & $\begin{array}{l}\text { Gestión integrada e inteligente de destinos turísticos } \\
\text { y información geográfica, mejora de la calidad } \\
\text { provincia Manabí - Ecuador. }\end{array}$ \\
\hline $\begin{array}{l}\text { 3- Fundamentación: problema y teorías a las que } \\
\text { responde }\end{array}$ & Conceptualización teórico metodológica. \\
\hline 4- Elementos que intervienen en su estructura & $\begin{array}{l}\text { Pasos o fases a seguir en la instrumentación de los } \\
\text { métodos, técnicas, medios y procedimientos. }\end{array}$ \\
\hline
\end{tabular}

Fuente: Elaboración propia.

La presente investigación utiliza antecedentes metodológicos ya citados de la Secretaria de Estado de Turismo de España respecto el Sistema Integral de Calidad Turística Española (SICTED) y de los Destinos Turísticos Inteligentes de la Sociedad Estatal para la Gestión de la Innovación y las Tecnologías Turísticas, S.A. (SEGITTUR). Aunque los escenarios son totalmente diferentes, se toman aquellos aspectos clave que fluyen en el contexto de Manabí, Ecuador. La metodología a emplear (Figura 5) tiene como esencia el carácter experimental trasformador y el enfoque inductivo, dado que parte de la decisión voluntaria de los empresarios para someterse al experimento y transformar los estilos de gestión. Con una visión integrada e inteligente, enfocada hacia la mejora de la calidad, se irán enrolando otras empresas y comunidades que lo soliciten. Bajo la orientación y supervisión de los gobiernos locales y el rol instructivo en entrenamiento personalizado de la 
Academia, se irán modificando los modos de actuación.

Las otras dos metodologías que se sistematizan en esta propuesta son Font (2002) que reconfigurada, expresa el cómo abordar el sistema turístico en el ámbito de la información geográfica: espacios, oferta, demanda, operadores turísticos. De Font, Blanco y Parra (2016) se obtiene el procedimiento de elaboración de los manuales de procedimientos, apelando a una secuencia de pasos, que parte de una declaración de intenciones, el mapa de proceso según tipo de empresa, el diagnóstico de intereses, desconocimientos, problemas y procesos, más las sugerencias de buenas prácticas para la mejora de la calidad. En la medida que los involucrados innovan e incorporan las TIC a sus productos, ofertas y experiencias; pasan a engrosar el equipo de entrenadores y a estimular la incorporación de nuevos voluntarios, revirtiéndose en una mejora homogénea del destino o territorio turístico.

Figura 5. Metodología propuesta.

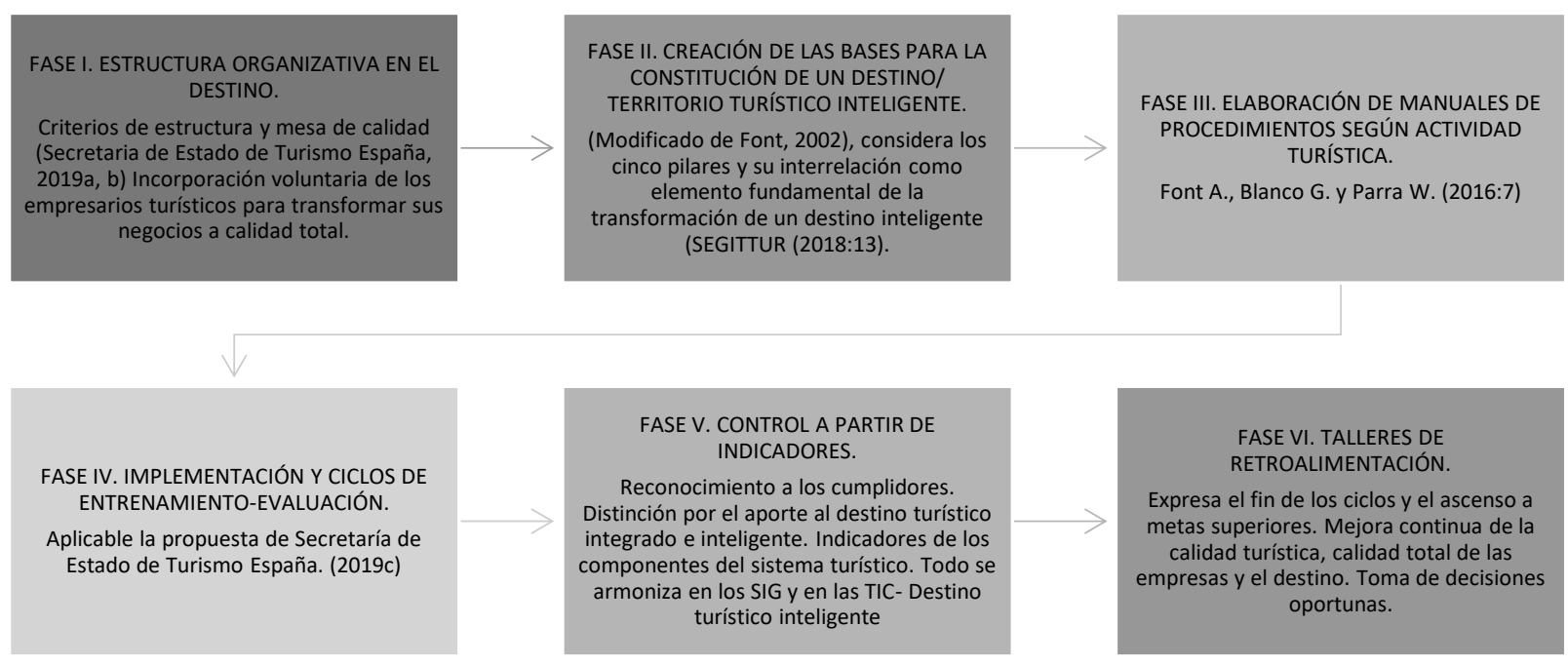

Fuente: Elaboración propia.

\section{CONCLUSIONES}

Los resultados del trabajo, muestran una comprensión sintetizada, ilustrada y modelada de los diferentes aspectos relacionados con la gestión integrada e inteligente en el turismo. Enfatiza en la derivación desde la connotación espacial/territorial de la actividad y sus atributos, hasta las formas y pilares de las organizaciones de gestión de destinos enfocados en la mejora de la calidad.

El estudio comparativo de otras metodologías implantadas para I a gestión turística integrada e inteligente, facilitan una concepción y la estructuración de una secuencia de fases factibles de aplicar en el contexto ecuatoriano de la provincia de Manabí. Incorpora como ejes claves la participación, las alianzas, las tecnologías y los sistemas de información geográfica; aspectos que inciden en la mejora de la calidad y la satisfacción a partir de toma de decisiones certeras y oportunas.

La metodología propuesta transita por seis fases principales: conformación de una organización y la incorporación voluntaria de empresarios y comunidades, creación de los pilares y estructuración de información con carácter geográfica, elaboración de manuales de procedimientos de buenas prácticas, implementación de ciclos de entrenamientos, reconocimiento a las mejoras de calidad, registro de indicadores en tecnologías de información, con énfasis en las geográficas, fin de ciclos y ascenso a metas superiores.

Los problemas identificados sobre la gestión turística en la provincia de Manabí y por tanto para los cantones que la componen, pueden ser mitigados o solucionados si se aplica la metodología 
propuesta para la gestión integrada e inteligente de destinos turísticos en el territorio en cuestión.

\section{REFERENCIAS}

Afnarius, S., Syukur, M., Ekaputra, E. G., Parawita, Y., \& Darman, R. (2020). Development of GIS for buildings in the customary village of minangkabau koto gadang, west Sumatra, Indonesia. ISPRS International Journal of GeoInformation, 9(6) doi:10.3390/ijgi9060365

Álvarez García J., Del Río Rama M., Durán Sánchez A., Urbano Orgaz B. (2017). Sistema de calidad turístico español: revisión de la literatura. Revista Ágora. Santa Cruz do Sul, v.19, n. 02, p. 0413, jul./dez. 2017.

Barreras Hernández, F. (2004). Los resultados de investigación en el área educacional. Conferencia presentada en el Centro de Estudios del ISP "Juan Marinello". Matanzas, Cuba.

Celdrán-Bernabeu, M. A., Mazón, J. -., \& Sánchez, D. G. (2018). Open data and tourism. implications for tourism management in smart cities and smart tourism destinationss. [Open Data y turismo. Implicaciones para la gestión turística en ciudades y destinos turísticos inteligentes*] Investigaciones Turísticas, (15), 49-78. doi:10.14198/INTURI2018.15.03

Corral-Marfil, J. A. (2012). La implantación del sistema español de calidad turística en destinos (SICTED): un caso práctico de gestión de destinos. Revista TuryDes. Vol 5, № 13 (Diciembre/Dezembro 2012). Málaga.

Crespo, Á. H., Gutiérrez, H. S. M., \& Sánchez, M. M. G. S. (2019). Smart services and equity of smart tourism destinations: Analysis from the perspective of the residents. [Servicios "smart" y valor de los destinos turísticos inteligentes: Análisis desde la perspectiva de los residentes] Investigaciones Regionales, 2019(45), 77-91. Retrieved from www.scopus.com

Dolan, R., Seo, Y., \& Kemper, J. (2019). Complaining practices on social media in tourism: A value co-creation and co-destruction perspective. Tourism Management, 73, 35-45. doi:10.1016/j.tourman.2019.01.017.

Font Aranda, M. 2002. Diferenciación geográfico ambiental del municipio y la ciudad de Santa Clara para la vigilancia en salud. Tesis presentada en opción al título de Doctor en Ciencias Geográficas. Universidad de La Habana. La Habana. Disponible en:http://repositorio.geotech.cu/jspui/bitstream/1 234/311/4/Diferenciaci\%C3\%B3n\%20geogr\%C3\%A 1fica\%20ambiental\%20del\%20municipio\%20y\%20l a\%20ciudad Parte1.pdf

Font A., Blanco G. y Parra W. 2016. Lineamientos metodológicos para la elaboración de manuales de procedimientos sobre gestión por procesos en alojamientos turísticos. $V$ Evento Internacional la Universidad en el Siglo XXI. octubre 2016. ISBN: 9789942-773-01-2. Disponible en: http://sigloxxi.espam.edu.ec/Ponencias/ $/$ ponenci as/5.pdf

Gao, F., \& Li, L. (2020). Design of smart travel management system based on cloud service. Paper presented at the Journal of Physics: Conference Series, , 1533(3) doi:10.1088/17426596/1533/3/032092 Retrieved from

\section{www.scopus.com}

García-Milon, A., Juaneda-Ayensa, E., OlartePascual, C., \& Pelegrín-Borondo, J. (2020). Towards the smart tourism destination: Key factors in information source use on the tourist shopping journey. Tourism Management Perspectives, 36 doi:10.1016/j.tmp.2020.100730

Gómez-Oliva, A., Alvarado-Uribe, J., ParraMeroño, M. C., \& Jara, A. J. (2019). Transforming communication channels to the co-creation and diffusion of intangible heritage in smart tourism destination: Creation and testing in ceutí (spain). Sustainability (Switzerland), 11(14) doi:10.3390/su11143848

Ivars-Baidal, J. A., \& Vera Rebollo, J. F. (2019). Planificación turística en España. De los paradigmas tradicionales a los nuevos enfoques: planificación turística inteligente. Boletín de la Asociación de Geógrafos Españoles, 82, 2765, 1-31. http://dx.doi.org/10.21138/bage.2765.

Ivars-Baidal, J. A., Celdrán-Bernabeu, M. A., Mazón, J.-N., \& Perles-Ivars, Á. F. (2017). Smart destinations and the evolution of ICTs: a new scenario for destination management? Current Issues in Tourism, 1-20. doi:10.1080/13683500.2017.1388771

Kiatkawsin, K., Sutherland, I., \& Lee, S. K. (2020). Determinants of smart tourist environmentally 
responsible behavior using an extended normactivation model. Sustainability (Switzerland), 12(12) doi:10.3390/SU12124934

Liberato, P., Alen, E., \& Liberato, D. (2018). Smart tourism destination triggers consumer experience: The case of porto. European Journal of Management and Business Economics, 27(1), 6-25. doi:10.1108/EJMBE-11-2017-0051

López de Ávila A., Lancis E., García S., Alcantud A., García B., Muñoz N. (2015). Informe destinos turísticos inteligentes: construyendo el futuro. Pautas de actuación para la conversión en destino turístico inteligente: Guía práctica. 207pp. Secretaría de Estado de Telecomunicaciones y para la Sociedad de la Información a la Sociedad Estatal para la Gestión de la Innovación y las Tecnologías Turísticas, S.A. (SEGITTUR). Madrid, septiembre de 2015.

Luis del Campo, V., Arribas Serrano, N., \& Morenas Martín, J. (2017). Quality in active tourism services in extremadura. [Calidad en los servicios de turismo activo de Extremadura] Apunts. Educación Física y Deportes, (129), 95-107. doi:10.5672/apunts.2014-0983.es.(2017/3).129.07

Organización Mundial del Turismo (2015), Manual práctico de gestión integral de la calidad de los destinos turísticos - Conceptos, implementación y herramientas para autoridades, instituciones y gestores de destinos, OMT, Madrid.

Organización Mundial del Turismo (OMT). (2019a). Definiciones de turismo de la OMT. OMT, Madrid, https://doi.org/10.18111/9789284420858

Organización Mundial del Turismo. (2019b). Directrices de la OMT para el fortalecimiento de las organizaciones de gestión de destinos (OGD) Preparando las OGD de cara a nuevos retos, OMT, Madrid, https://doi.org/10.18111/9789284420933

Pai, C. -., Liu, Y., Kang, S., \& Dai, A. (2020). The role of perceived smart tourism technology experience for tourist satisfaction, happiness and revisit intention. Sustainability (Switzerland), 12(16) doi:10.3390/su12166592

Parasuraman, A., Zeithaml, V. A., \& Berry, L. L. (1988). SERVQUAL: a multiple-item scale for measuring consumer perceptions of service quality. Journal of Retailing, 64(1), 12 - 40.
Proaño Ponce, W. P. y Ramírez Pérez, J. F. (2017). Modelo de Desarrollo Turístico Sostenible para cantones costeros: herramienta para actores locales de Manabí, Ecuador Revista Posgrado y Sociedad. Sistema de Estudios de Posgrado. Universidad Estatal a Distancia. ISSN: 2215-2172 Volumen 15, Número 2, 2017, 65-78. PP. 72 RevistaSEP-PyS@uned.ac.cr

Ranasinghe, J. P. R. C., Danthanarayana, C. P., Ranaweera, R. A. A. K., \& Idroos, A. A. (2020). Role of destination smartness in shaping tourist satisfaction: A SEM based on technological attributes in sri lanka. Paper presented at the IOP Conference Series: Earth and Environmental Science, , 511(1) doi:10.1088/17551315/511/1/012001 Retrieved from www.scopus.com

Salessi M. L. (2017). Destinos Turísticos Inteligentes: Una perspectiva desde la inclusión social y la participación comunitaria. Actas del Seminario Internacional Destinos Turísticos Inteligentes: nuevos horizontes en la investigación y gestión del turismo. Universidad de Alicante, 26 y 27 de octubre de 2017. Pp. 215. Consultado 18 de octubre de $2019 . \quad$ Recuperado de: https://rua.ua.es/dspace/bitstream/10045/70141/ 5/Actas-Seminario-Destinos-TuristicosInteligentes.pdf

Secretaría de Estado de Turismo - SEGITTUR. (2018). La estrategia española de destinos turísticos inteligentes. Jornadas Europeas, sostenibilidad y territorios inteligentes, 5 de abril de 2018. Cáceres, España. Consultado: 18 de octubre de 2019. Recuperado de: https://www.dipcaceres.es/comun/galerias/galeriaDescargas/cacer es/desarrollolocal/03 Estrategia Destinos Tcos In teligentes.pdf

Secretaría de Estado de Turismo. (2018). La estrategia española de destinos turísticos inteligentes. Consultado 18 de octubre de 2019. Recuperado de: https://docplayer.es/95511513-Laestrategia-espanola-de-destinos-turisticosinteligentes.html

Secretaría de Estado de Turismo. (2019a). ¿Qué es el SICTED? Sistema Integral de Calidad Turística en Destinos. Octubre de 2019 Ministerio de Industria, Energía y Turismo. Licencia: Creative Commons Reconocimiento 2.0 España. Madrid. Consultado 18 de octubre de 2019. Recuperado de: 
https://www.calidadendestino.es/documentos/20 191003 Que es el SICTED.pdf

Secretaría de Estado de Turismo. (2019b). Estructura organizativa del Sistema Integral de Calidad Turística Española en Destinos (SICTED). Consultado 18 de octubre de 2019. Recuperado de: https://www.calidadendestino.es/documentos/20 190408 Estructura del SICTED.pdf

Secretaría de Estado de Turismo. (2019c). Metodología de Manuales de Buenas Prácticas (MBP) Versión 02, ene.-19. Ministerio de Industria, Energía y Turismo. Licencia: Creative Commons Reconocimiento 2.0 España. Madrid. Consultado 18 de octubre de 2019. Recuperado de: https://sictedvalenciacom.files.wordpress.com/20 19/06/metodologc3adademanualesdebuenasprc3 a1cticasv220190109.pdf

SENPLADES. (2019). Agendas Zonales. Zona 4 Pacífico, Manabí - Santo Domingo de los Tsáchilas. Primera Edición, 2019. Senplades, abril 2019. Barbasquillo Km 51/2 vía a San Mateo. Manta, Ecuador. www.planificacion.gob.ec
Shen, S., Sotiriadis, M., \& Zhang, Y. (2020). The influence of smart technologies on customer journey in tourist attractions within the smart tourism management framework. Sustainability (Switzerland), 12(10) doi:10.3390/su12104157

Sigalat-Signes, E., Calvo-Palomares, R., RoigMerino, B., \& García-Adán, I. (2020). Transition towards a tourist innovation model: The smart tourism destination: Reality or territorial marketing? Journal of Innovation and Knowledge, 5(2), 96-104. doi:10.1016/j.jik.2019.06.002

Su, L., Swanson, S. R., \& Chen, X. (2016). The effects of perceived service quality on repurchase intentions and subjective well-being of chinese tourists: The mediating role of relationship quality. Tourism Management, 52, 82-95. doi:10.1016/j.tourman.2015.06.012 